\title{
A Coupled Lagrangian-Eulerian Scheme for Reacting Flow Modeling
}

\author{
H. N. Najm, \\ Sandia National Laboratories, Livermore, CA, USA. \\ hnnajm@ca.sandia.gov \\ R. B. Milne, \\ Lawrence Berkeley National Laboratory, Berkeley, CA, USA. \\ K. D. Devine, and S. N. Kempka \\ Sandia National Laboratories, Albuquerque, NM, USA.
}

\section{Abstract}

We describe a coupled Lagrangian-Eulerian numerical scheme for modeling reacting flow in two dimensions. The scheme uses the Lagrangian vortex method for solving the Navier-Stokes equations, and an Eulerian finite difference implementation on a multi-layered adaptive mesh for the scalar conservation equations. Coupling terms involving baroclinic and diffusional circulation source terms as well as expansion sources for dilatation resulting from heat release are implemented as necessary. A boundary element method is used for the potential flow solution. A fast multipole method is used for all velocity field evaluations. The numerical construction is presented along with representative buoyant reacting jet flow results.

\section{Introduction}

The detailed modeling of chemically reacting flow requires excessive spatial resolution in order to adequately describe internal flame structure. Equally fine temporal resolution is necessary for the time integration of stiff chemical kinetic mechanisms. The present work is concerned with the development of a numerical scheme for addressing the spatial resolution issue.

The use of a purely Lagrangian construction would incorporate an implicitly adaptive set of computational elements, providing efficient spatial resolution of flow/flame structures. On the other hand, this approach is complicated by the need for spatially varying species-specific transport properties in the flame region. Alternatively, Eulerian finite difference schemes with adaptive mesh refinement (AMR) have been used to model hyperbolic systems with propagating fronts [1], and low Mach number reacting flow [2]. However, the need for a Poisson equation solution for the pressure field in the low Mach number formulation necessitates an iterative construction, which leads to scalability concerns on massively parallel hardware.

In order to combine the best aspects of the above two approaches, we developed a coupled Lagrangian-Eulerian scheme [3], where the vortex method is used to solve the momentum equations and a finite difference AMR scheme is used to solve the scalar conservation equations in two dimensions (2D). The result is an efficient explicit scheme for reacting flow modeling. This scheme was implemented in a distributed parallel code with dynamic load balancing. It has been used to study the structure and dynamics of a lifted buoyant jet diffusion flame. 


\section{$3 \quad$ Numerical Methodology}

We assume a 2D flow and employ the low Mach number approximation. Since the domain of interest is open, the stagnation pressure $p_{o}[4]$ is assumed constant. Other assumptions are outlined below.

\subsection{Model Formulation}

The continuity equation is written in the conservative form: $\partial \rho / \partial t+\nabla \cdot(\rho \mathbf{v})=0$, where $\mathbf{v}=(u, v)$ is the velocity vector, and $\rho$ is the density. The energy equation is written as:

$$
\frac{\partial T}{\partial t}=-\mathbf{v} \cdot \nabla T+\frac{1}{\operatorname{RePr}} \frac{\nabla \cdot(\lambda \nabla T)}{\rho c_{p}}+\frac{1}{\operatorname{ReSc}} \frac{\mathbf{Z} \cdot \nabla T}{c_{p}}+D a \frac{w_{T}}{\rho c_{p}}
$$

where $\mathbf{Z}=\sum_{i=1}^{N} c_{p, i} D_{i N} \nabla Y_{i}, w_{T}=-\sum_{i=1}^{N} h_{i} w_{i}, T$ is temperature, $c_{p}$ is the mixture specific heat at constant pressure, $\lambda$ is the mixture thermal conductivity, $D a$ is the Damköhler number, $h_{i}$ is the enthalpy of species $i, w_{i}$ is the chemical rate of production/consumption of species $i, \operatorname{Re}, \operatorname{Pr}$, $S c$ are the Reynolds, Prandtl, and Schmidt numbers respectively, and $Y_{i}$ is the mass fraction of species $i$.

We note that transport properties of the gas mixture $(\mu, \lambda)$ are in general complicated functions of $T$, and $Y_{i}, i=1, \ldots, N$. Practically, since $\mathrm{N}_{2}$ is a dominant species everywhere in the mixtures under consideration, we use $(\mu, \lambda)=(\mu, \lambda)_{\mathrm{N}_{2}}(T)$. Similarly, we approximate the mixture-averaged mass diffusion coefficients by the binary mass diffusion coefficients into $\mathrm{N}_{2}$ at the local temperature. By convention, we set the dominant species in the mixture as the $N$-th species, hence the use of $D_{i N}$ above for the diffusion coefficient of the $i$-th species into the mixture. Finally, thermodynamic properties $\left(c_{p, i}, h_{i}\right)$ are empirically known functions of $T$, and $c_{p}=\sum Y_{i} c_{p, i}$.

The species conservation equation, is written as:

$$
\frac{\partial Y_{i}}{\partial t}=-\mathbf{v} \cdot \nabla Y_{i}+\frac{1}{R e S c} \frac{\nabla \cdot\left(\rho D_{i N} \nabla Y_{i}\right)}{\rho}+D a \frac{w_{i}}{\rho}
$$

In order to obtain an evolution equation for vorticity $(\omega=\nabla \times \mathbf{v})$, the curl operator is applied to the momentum equations. After some manipulation, we get (using $(\omega \cdot \nabla) \mathbf{v}=0$ for a $2 \mathrm{D}$ flow):

$$
\frac{\partial \omega}{\partial t}+(\mathbf{v} \cdot \nabla) \omega+\omega(\nabla \cdot \mathbf{v})=\frac{1}{\rho} \nabla \rho \times\left(\frac{1}{F r} \mathbf{g}-\frac{D \mathbf{v}}{D t}\right)+\frac{1}{R e} \frac{\nabla \times \mathbf{\Phi}}{\rho} .
$$

where $\mathbf{g}$ is the acceleration vector due to gravity, and $\mathbf{\Phi}$ is the divergence of the deviatoric stress tensor, which is given in indicial notation by

$$
\boldsymbol{\Phi}=\frac{\partial \tau_{j i}}{\partial x_{j}}=\frac{\partial}{\partial x_{j}}\left[-\frac{2}{3} \mu \delta_{i j} \frac{\partial u_{k}}{\partial x_{k}}+\mu\left(\frac{\partial u_{i}}{\partial x_{j}}+\frac{\partial u_{j}}{\partial x_{i}}\right)\right] .
$$

From this, the evolution of circulation for a fluid area $A$ is found to be,

$$
\frac{d}{d t} \int_{A} \omega \cdot \mathbf{n} d A=\int_{A} d \mathbf{A} \cdot\left[\frac{1}{\rho} \nabla \rho \times\left(\frac{1}{F r} \mathbf{g}-\frac{D \mathbf{v}}{D t}\right)+\frac{1}{R e} \frac{\nabla \times \Phi}{\rho}\right] .
$$

The viscous term of the vorticity transport equation, $(\nabla \times \Phi) / \rho$, can be expressed simply as $\nu \nabla^{2} \omega$, if the fluid viscosity is spatially uniform and the flow is divergence free. Neither of these 
conditions is applicable for the reacting flow of interest, and there is no significant simplification from the above form. The complexity of this term essentially precludes a Lagrangian approach at the present time. Thus, it is evaluated with a finite difference discretization using the same grid as for scalar transport.

In the context of a vorticity formulation, the velocity field must be calculated from the vorticity field. The velocity divergence due to combustion heat release, and the potential velocity field dictated by the domain boundaries, must also be included in calculating the velocity field. The approach used here is based on the well-known kinematic Helmholtz decomposition of a velocity field into additive contributions from the curl of the velocity field (the vorticity field), the divergence of the velocity field, and the normal velocity boundary condition [5], $\mathbf{v}=\mathbf{v}_{\omega}+\mathbf{v}_{D}+\mathbf{v}_{p}$, where $\mathbf{v}_{\omega}$ is the velocity induced by the vorticity field in the unbounded simply-connected domain $R_{\infty}, \mathbf{v}_{D}$ is the velocity due to local expansion resulting from reaction heat release rate (and transport),

$$
D=\nabla \cdot \mathbf{v}=\frac{1}{T} \frac{D T}{D t}+\bar{W} \sum_{i=1}^{N} \frac{1}{W_{i}} \frac{D Y_{i}}{D t}
$$

in the infinite domain, and $\mathbf{v}_{p}$ is a divergence-free irrotational velocity field which is represented as the gradient of a potential function, $\mathbf{v}_{p}=\nabla \phi$, with $\nabla^{2} \phi=0$ and appropriate boundary conditions.

\subsection{Numerical Scheme}

\subsubsection{Adaptive Mesh Refinement}

In recent years, adaptive methods for solving partial differential equations have been growing in popularity [6]. Early adaptive techniques of mesh motion have given way to methods that combine mesh refinement/coarsening with order variation [6]. In this regard, adaptivity for partial differential equations is following the path of similar strategies used for ordinary differential equations [7].

In this work, we have enhanced our Eulerian method for the solution of the scalar transport equations with an adaptive mesh-refinement strategy. With adaptive mesh refinement, a spatial error estimate is used to determine which regions of the computational domain need greater resolution to maintain the solution's estimated error below a user-defined tolerance. Cells in high-error regions are subdivided into finer cells. A local time stepping strategy is used [8]; that is, refinement is performed in both space and time to maintain stable convective and viscous CFL numbers on refined meshes. The solution is obtained on each mesh level recursively, with smaller time steps being taken on finer meshes.

The present adaptive mesh refinement code is based on that of Devine and Flaherty [1]. Their code used a local finite element method with adaptive mesh and order enrichment to solve hyperbolic systems on 2D uniform meshes. While our numerical approach differs from theirs, we were able to use their underlying data structures, parallel mesh refinement implementation, dynamic loadbalancing algorithm, and data migration strategies to reduce the code development effort.

\subsubsection{Eulerian Discretization of the Scalar Conservation Equations}

The energy equation and $N-1$ scalar equations are discretized on the Eulerian multi-layered adaptive mesh, using second-order difference formulations. Diffusive terms are discretized using 
centered second-order differences. The convective terms are discretized using a second-order Godunov upwind scheme $[9,10]$, resulting in a convective scheme that is stable to arbitrary grid Reynolds or Peclet numbers. The Pe number stability characteristic of this construction is necessary for stable time integration of the scalar equations on the coarsest mesh level, with largest $P e$. Given these spatial discretizations, the resulting computational stencil at cell $(i, j)$ spans the nine cells $\{([i-2, i+2], j),(i,[j-2, j+2])\}$. Further, the corner cells $(i \pm 1, j \pm 1)$ are needed for numerical interpolations, such that the overall necessary stencil includes 13 points.

On any particular mesh level, the spatial discretization is based on a uniform mesh in both directions. In order to avoid cumbersome conditions on derivative discretizations at mesh boundaries, both internal and at the domain edges, two rows of redundant cells are created around each mesh patch, extending beyond each mesh boundary. Scalar and velocity values at these cells are found by interpolation from their coarser-mesh parents and neighbors. Thus the discretization of the scalar conservation equations is concerned only with uniform rectangular meshes.

\subsubsection{Lagrangian Discretization of the Vorticity Transport Equation}

The vorticity field $\omega(x, y)$ is discretized using Lagrangian vortex elements, such that $\omega(x, y) \approx$ $\sum_{k} \Gamma_{k} f_{\delta}\left(r_{k} / \delta\right)$ where $\Gamma_{k}$ is the circulation strength assigned to element $k, r_{k}=\left|\mathbf{x}-\mathbf{x}_{k}\right|, \delta$ is the element core radius, and $f_{\delta}$ is a smooth compact core function with $\int f_{\delta}(r) d r=1$. We use a second-order gaussian core function, $f_{\delta}(r)=\left(1 / \pi \delta^{2}\right) \exp \left(-r^{2}\right)$.

Given a vorticity field $w(x, y)$, this discretization is accomplished using an underlying uniform mesh of cell size $h_{\omega} \times h_{\omega}$, such that an element is created at the center of cell $\left(x_{k}, y_{k}\right)$ with strength $\Gamma_{k}=\omega\left(x_{k}, y_{k}\right) h_{\omega}^{2}$, when $\Gamma_{k}$ is above a given threshold. The core radius is chosen to provide necessary overlap [11], with $\delta=h_{\omega}^{0.9}$. This procedure is used both at $t=0$ to discretize the initial vorticity field, and for creation of new elements when necessary due to baroclinic and viscous circulation source terms. This construction allows the use of the same core radius for all vortex elements. On the other hand, it can lead to large numbers of vortices, which necessitates frequent redistribution of the vortex elements as indicated below.

The dilatation/expansion field $D(x, y)$ is evaluated as indicated above at each leaf-cell center on the adaptive mesh. This field is discretized using expansion source elements created on a uniform mesh with $h_{D} \times h_{D}$ cells, with strengths $\epsilon_{k}=D\left(x_{k}, y_{k}\right) h_{D}^{2}$, and core functions $g_{\delta}$, such that $D(x, y) \approx \sum_{k} \epsilon_{k} g_{\delta}\left(r_{k} / \delta\right)$, where we use the same gaussian core function as for the vortices.

The evaluation of $\mathbf{v}_{\omega}(x, y)$ from the vortex element distribution uses

$$
\mathbf{v}_{\omega}=\sum_{k}-\frac{\Gamma_{k}}{2 \pi} \frac{\left[\left(y-y_{k}\right),-\left(x-x_{k}\right)\right]}{r_{k}^{2}}\left(1-e^{-r_{k}^{2} / \delta^{2}}\right) .
$$

where the summation is over all vortex elements. Similarly $\mathbf{v}_{D}$ is found from a similar discretization, summing over all expansion sources,

$$
\mathbf{v}_{D}=\sum_{k} \frac{\epsilon_{k}}{2 \pi} \frac{\left[\left(x-x_{k}\right),\left(y-y_{k}\right)\right]}{r_{k}^{2}}\left(1-e^{-r_{k}^{2} / \delta^{2}}\right) .
$$

The potential flow solution required to impose boundary conditions and provide $\mathbf{v}_{p}=\nabla \phi$ is computed using the Boundary Element Method. An adaptive Fast Multipole Method [12] is used to compute velocities induced by vortex and boundary elements as well as expansion sources. The 
velocity field is computed at the centers of vortex elements, to be used in advecting them, and at the Eulerian mesh cell walls, to be used in the convection of scalars and the computation of baroclinic and diffusion source terms. Boundary conditions for the jet flow modeled here include inflow at the bottom boundary, slip vertical walls, and an outflow top edge. At the inflow boundary, vortex elements are injected into the domain with strengths corresponding to the circulation convected across the domain edge over $h_{\omega}$-wide discrete edge-segments in each time step, given a specified inflow velocity profile. Vortex elements are deleted when they cross the outflow edge.

\subsubsection{Redistribution of Vortex Elements}

It is well established that the long time accuracy of vortex method solutions to the momentum equations suffers due to the increasing irregularity of the Lagrangian "mesh" composed of element locations $[13,14]$. Inherent in the construction of the method, elements move according to the local velocity field, and may therefore become concentrated in certain regions and depleted elsewhere. As elements move apart, the loss of overlap between nearest elements' cores leads to loss of accuracy. A useful remedy is a periodic remeshing or redistribution [15] of the vortex elements onto a regular mesh using an appropriate interpolation kernel. This is also useful in terms of reducing the number of elements in the domain, which in the present flow increases substantially due to the creation of new elements by baroclinic and viscous circulation source terms. On the other hand, redistribution can introduce perturbations to the original vorticity field, depending on the smoothness of the interpolation kernel. In particular, the present scheme requires the evaluation of the viscous term in the vorticity transport equation from the velocity field available at mesh points. This involves third order derivatives of the velocity field, and is therefore sensitive to minor noise in the velocity. As a consequence, we find that the discontinous $\Lambda_{2}$ kernel $[15,16]$ is inadequate in the present context, because of resulting spurious noise in the viscous source terms. Rather, the smoother $W_{4}$ kernel [17] was necessary to maintain smoothness of the source terms after redistribution.

\subsection{Coupled Lagrangian-Eulerian Time Integration}

A coupled integration approach is constructed based on a second-order Runge-Kutta/predictorcorrector formulation for both the Eulerian and Lagrangian integrations. The integration procedure is as follows.

1. Assume starting at time level $t^{n}$, with all flow quantities known on all mesh levels. There are $N_{v}$ vortex elements $\omega_{i}=\left(\chi_{i}, \Gamma_{i}\right), i=1,2, \ldots, N_{v}$, at lagrangian locations $\chi_{i}$ and with circulation strengths $\Gamma_{i}$. There are $N_{P}$ boundary elements $P_{j}=\left(\pi_{j}\right), j=1,2, \ldots, N_{P}$, at locations $\pi_{j}$, and with appropriate strengths. There are also $N_{e}$ expansion sources $S_{k}=$ $\left(s_{k}, \epsilon_{k}\right), k=1,2, \ldots, N_{e}$, at locations $s_{k}$ and with strengths $\epsilon_{k}$, based on $D_{i j}=(\nabla \cdot \mathbf{v})_{i j}$ at $t^{n}$. We also have known velocity field values computed from this distribution of vortices, boundary elements, and sources, at all vortex element locations $\left(\mathbf{v}_{i}^{n}\right)$ and at all grid locations $\left(\mathbf{v}_{i j}^{n}\right)$, for all mesh levels. The baroclinic $B_{i j}^{n}$ and diffusional $\mathcal{V}_{i j}^{n}$ circulation source/sink terms are also known at all cell centers. We also have scalar values at cell centers on all meshes, $c_{i j}^{n}$, where $c=\left\{c_{k}\right\}=\left(T, \rho, Y_{1}, Y_{2}, \ldots, Y_{N}\right)$.

2. Propagate the vorticity field, predictor, from $t^{n}$ to $t^{n+1}=t^{n}+\Delta t_{L}$.

$$
\tilde{\chi}_{i}^{n+1}=\chi_{i}^{n}+\mathbf{v}_{i}^{n} \Delta t_{L} \quad\left(i=1,2, \ldots, N_{v}\right)
$$

ESAIM: Proc., Vol. 7, 1999, $308-313$ 
where $\Delta t_{L}$ is the Lagrangian time step. This involves adding elements at the domain inlet, and elsewhere in the domain if necessary, due to diffusion and baroclinicity. The circulation change in each mesh cell is given by,

$$
\Delta \Gamma_{i j}=\Delta t_{L}\left(B_{i j}^{n}+\mathcal{V}_{i j}^{n}\right)
$$

This circulation, when above threshold, is used to create new elements at convected leaf-cell centers. Vortex elements added in this (predictor) step will be used to compute velocity fields and then discarded. The corrected $\tilde{B}_{i j}^{n+1}$ and $\tilde{D}_{i j}^{n+1}$, along with the boundary conditions, are then used to add new permanent elements, or remove elements that leave the domain, in the corrector step below.

3. Propagate scalar fields, predictor, on all grids, holding the grid velocity field constant at $\mathbf{v}_{i j}^{n}$. In general, $\Delta t_{L}=m \Delta t_{E}^{o}$, where $\Delta t_{E}^{o}$ is the Eulerian time step on mesh 0 , and $m$ is a positive integer. Thus, for any scalar $c(\mathbf{x}, t)$, perform the time integration on all grids, following the recursive AMR integration procedure, from $t^{n}$ to $t^{n}+\Delta t_{L}$. Generally, for $\Delta t_{L}=K \Delta t_{E}^{l}\left(\Delta t_{E}^{l}\right.$ is the Eulerian time step on mesh level $l$ ), for $k=1,2, \ldots, K$ :

$$
\tilde{c}^{n+k / K}=\tilde{c}^{n+\frac{k-1}{K}}+\Delta t_{E}^{l} g_{c}\left(\mathbf{v}^{n}, \tilde{c}^{n+\frac{k-1}{K}}\right)
$$

where, $\partial c / \partial t=g_{c}(\mathbf{v}, c)$ is the right-hand-side of the conservation equation for $c$, and $\tilde{c}^{n}=c^{n}$.

4. Find $\tilde{\mathbf{v}}_{i}^{n+1}$, the new predicted velocity at the predicted new vortex element locations. Evaluate the velocity $\tilde{\mathbf{v}}_{i}^{n+1}$ at the center of each vortex element $\omega_{i}$, with $\tilde{\mathbf{v}}_{i}^{n+1}=\tilde{\mathbf{v}}^{n+1}\left(\tilde{\chi}_{i}^{n+1}\right)=$ $\tilde{\mathbf{v}}_{\omega, i}^{n+1}+\tilde{\mathbf{v}}_{p, i}^{n+1}+\tilde{\mathbf{v}}_{D, i}^{n+1}$. Here, $\mathbf{v}_{\omega}=\mathbf{v}_{\omega}(\underline{\omega})$, where $\underline{\omega}=\left\{\omega_{i}\right\}$ is the set of all vortex elements, $\mathbf{v}_{p}=\mathbf{v}_{p}(\underline{P})$, where $\underline{P}=\left\{P_{j}\right\}$ is the set of all boundary elements, and $\mathbf{v}_{D}=\mathbf{v}_{D}(\underline{S})$, where $\underline{S}=\left\{S_{k}\right\}$ is the set of all expansion sources. This involves the following internal steps:

- Using $\underline{\omega}$, find $\mathbf{v}_{\omega}$, in an unbounded infinite simply connected domain.

- Using the predicted scalar field on the adaptive mesh, evaluate $D_{i j}$ in each childless cell. Create expansion sources $S_{k}$ in cells with $D_{i j}$ above a certain threshold, and compute $\mathbf{v}_{D}$, in an unbounded infinite simply connected domain.

- Using the computed $\mathbf{v}_{\omega}$ and $\mathbf{v}_{D}$, enforce velocity boundary conditions to find boundary element field $\underline{P}$ strengths, and evaluate $\mathbf{v}_{p}$.

- Combine these velocity fields to get $\tilde{\mathbf{v}}_{i}^{n+1}=\tilde{\mathbf{v}}_{\omega, i}^{n+1}+\tilde{\mathbf{v}}_{p, i}^{n+1}+\tilde{\mathbf{v}}_{D, i}^{n+1}, i=1,2, \ldots, N_{v}$.

5. Evaluate the predicted velocity field $\tilde{\mathbf{v}}_{i j}^{n+1}$ on all grids at predicted time $\tilde{t}^{n+1}$, again using the contributions due to the vortical, potential, and dilatational components.

6. Evaluate the strength of baroclinic and diffusion circulation source terms for each cell at the new predicted time level, $\tilde{B}_{i j}^{n+1}$ and $\tilde{\mathcal{V}}_{i j}^{n+1}$, where the term $\partial \mathbf{v} / \partial t$ in $D \mathbf{v} / D t$ is evaluated using the fields : $\mathbf{v}_{i j}^{n}$ and $\tilde{\mathbf{v}}_{i j}^{n+1}$ on the grid. Note that the predicted field is $O\left(\Delta t_{L}\right)$; hence a first-order discretization for $\partial \mathbf{v} / \partial t$ is adequate here. Namely, $\left.\partial \widetilde{\mathbf{v} / \partial} t\right|_{i j} ^{n+1}=\left(\tilde{\mathbf{v}}_{i j}^{n+1}-\mathbf{v}_{i j}^{n}\right) / \Delta t_{L}$.

7. Propagate the vorticity field, corrector, from $t^{n}$ to $t^{n+1}=t^{n}+\Delta t_{L}$,

$$
\chi_{i}^{n+1}=\chi_{i}^{n}+\frac{\Delta t_{L}}{2}\left(\mathbf{v}_{i}^{n}+\tilde{\mathbf{v}}_{i}^{n+1}\right) \quad\left(i=1,2, \ldots, N_{v}\right)
$$

Also, add/remove elements (permanently) at domain inflow/outflow boundaries, and add necessary cicrculation in each leaf mesh cell due to baroclinic and diffusional sources.

$$
\Delta \Gamma_{i j}=\frac{\Delta t_{L}}{2}\left(B_{i j}^{n}+\mathcal{V}_{i j}^{n}+\tilde{B}_{i j}^{n+1}+\tilde{\mathcal{V}}_{i j}^{n+1}\right)
$$


8. Propagate scalar fields, sequence of predictor-correctors, using time-interpolated velocity field values on the grid, based on linear interpolation between $\mathbf{v}_{i j}^{n}$ and $\tilde{\mathbf{v}}_{i j}^{n+1}$. For $k=1,2, \ldots, K$ :

$$
\begin{aligned}
\tilde{c}^{n+k / K} & =c^{n+\frac{k-1}{K}}+\Delta t_{E}^{l} g_{c}\left(\hat{\mathbf{v}}^{n+\frac{k-1}{K}}, c^{n+\frac{k-1}{K}}\right) c^{n+k / K} \\
& =c^{n+\frac{k-1}{K}}+\frac{1}{2} \Delta t_{E}^{l}\left[g_{c}\left(\hat{\mathbf{v}}^{n+\frac{k-1}{K}}, c^{n+\frac{k-1}{K}}\right)+g_{c}\left(\hat{\mathbf{v}}^{n+k / K}, \tilde{c}^{n+k / K}\right)\right]
\end{aligned}
$$

where, $\hat{\mathbf{v}}^{n+k / K}=(k / K)\left(\tilde{\mathbf{v}}^{n+1}-\mathbf{v}^{n}\right)+\mathbf{v}^{n}$.

9. Repeat Step 4, evaluate new corrected velocities at vortex element centers, $\mathbf{v}_{i}^{n+1}$.

10. Repeat Step 5, evaluate new corrected velocities at grid cell walls, all meshes, $\mathbf{v}_{i j}^{n+1}$.

11. Evaluate the strength of baroclinic and diffusion circulation source terms for each cell at the new corrected time level $B_{i j}^{n+1}$ and $\mathcal{V}_{i j}^{n+1}$. Here a second-order discretization of $\partial \mathbf{v} / \partial t$ is necessary. Yet, we will only use two time levels, $\mathbf{v}^{n}$ and $\mathbf{v}^{n+1}$. The need for $\mathbf{v}^{n+1}$ is the main reason for doing Steps 9 and 10 at the end of a time step rather than at the beginning. A two-time-level second-order $\partial \mathbf{v} / \partial t$ discretization is given by: $\partial \mathbf{v} /\left.\partial t\right|^{n+1}=$ $\left(2 / \Delta t_{L}\right)\left(\mathbf{v}^{n+1}-\mathbf{v}^{n}\right)-\partial \mathbf{v} /\left.\partial t\right|^{n}$.

12. This is the end of the Lagrangian time step. An optional redistribution of the vortex element field is implemented here as indicated above, following the $W_{4}$ scheme in [17].

\section{Results and Discussion}

The time-convergence rate of the above scheme was studied using a simple flow case, a decaying hot vortex in an unbounded 2D domain, on a uniform mesh and with no redistribution of the vortex elements. We observe general second order convergence when the circulation source terms due to diffusion and baroclinicity are neglected (but including the expansion sources and other coupling terms). When vorticity diffusion is included, the convergence rate of the vorticity and viscous momentum source term fields (computed from the velocity fields on the Eulerian mesh) degrades to first order, while second-order convergence of other tems is maintained, as shown in Figure 1. Further, when baroclinic vorticity generation is allowed, first order convergence is observed the velocity field as well. The degradation of the convergence rate seems to be a result of the specific implementation for computing and injecting mesh-generated vorticity. More work is required to address this issue. Moreover, specific issues, such as the impact of vorticity redistribution and mesh refinement on time accuracy have yet to be studied.

The scheme has also been used to compute the interaction of a premixed methane-air flame with a counter rotating vortex-pair, using a global single-step chemical kinetic mechanism. Comparisons with the results from a finite difference computation [18] of the same flow field reveals very similar flow and flame development, along with the topology and structure of regions of baroclinic vorticity generation, velocity field divergence, and flame heat release rate.

We also used this scheme to study the flow and flame dynamics involved in a lifted laminar jet diffusion flame, with organized forcing of jet flow structures. We consider a $20 \times 20 \mathrm{~cm}$ rectangular domain with a planar jet of fuel, of width $1.16 \mathrm{~cm}$, centered at $x=10 \mathrm{~cm}$, flowing vertically upward. The fuel jet velocity is $0.8 \mathrm{~m} / \mathrm{s}$, and is surrounded by coflow air with a $0.1 \mathrm{~m} / \mathrm{s}$ velocity. The jet Reynolds number is 480, with a Prandtl number of 0.94, and a fuel-diffusion Schmidt number of 


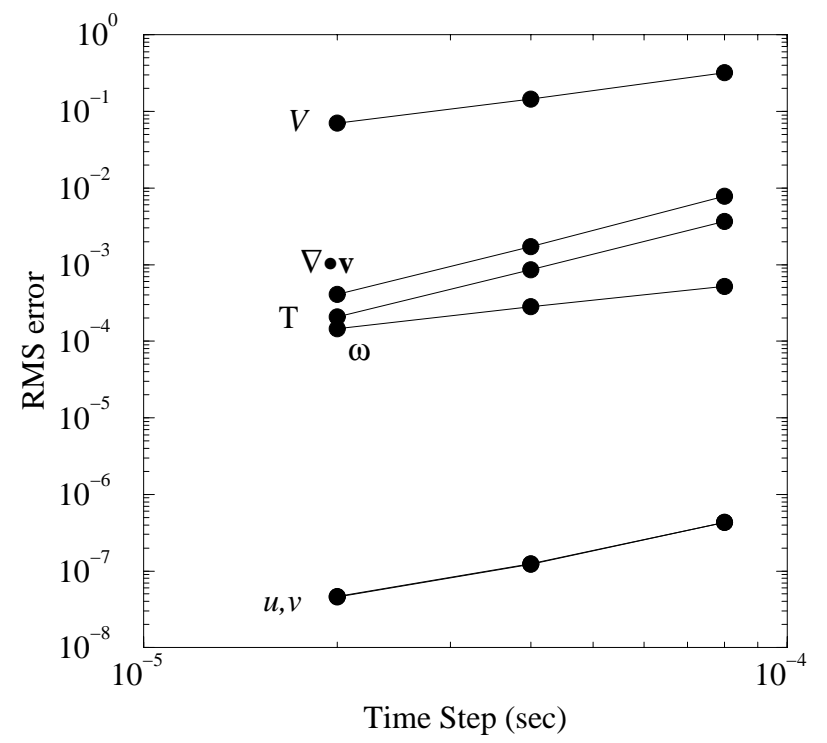

Figure 1: Time convergence of various flow quantities for a decaying hot vortex in an unbounded domain, using a uniform mesh, and neglecting baroclinic vorticity. Temperature $(T), u$ and $v$ velocities, and dilatation rate $(\nabla \cdot \mathbf{v})$ exhibit second-order convergence rate. On the other hand, vorticity $\omega$ and the viscous momentum source term $V=(\nabla \times \Phi) / \rho$ exhibit first-order convergence.

0.85. The jet flow is forced with an impulse function at a frequency of $7.5 \mathrm{~Hz}$, with a large forcing amplitude chosen to induce strong vortex structures in the vicinity of the flame base.

The composition of the fuel jet is $40 \% \mathrm{CH}_{4}, 60 \% \mathrm{~N}_{2}$ by volume. Both jet and coflow are at room temperature. We use a single-step irreversible global chemical mechanism : $\mathrm{CH}_{4}+2 \mathrm{O}_{2} \Rightarrow$ $\mathrm{CO}_{2}+2 \mathrm{H}_{2} \mathrm{O}$, with an Arrhenius forward rate, chosen to match global flame structure as computed with a detailed mechanism. The flowfield is initialized with global 1D flame profiles, which are arranged in 2D to correspond to two diffusion flames extending from the vicinity of the jet edges to the top of the domain. A global Lagrangian-Eulerian time step of $0.125 \mathrm{~ms}$ is used. Five levels of adaptive mesh refinement are used. The coarsest mesh, Mesh-0, is 30x30, with cell size $h_{0}=0.67 \mathrm{~cm}$. The finest mesh (Mesh-5) cell size is $h_{5}=h_{0} / 2^{5}$. Vortex elements are created with center separation $h_{\omega}=h_{4}$, with $\delta=0.057 \mathrm{~cm}$, and are redistributed every 5 time steps. The average number of vortex elements increases from around $10^{5}$ to $1.6 \times 10^{5}$ over the duration of the present run.

As the flow evolves in time, the adaptive mesh structure changes accordingly to maintain adequate spatial resolution. The Mesh-5 region corresponds generally to the steep shear layer at the jet exit and the base of the lifted flame, where peak heat release rate is observed.

The flame base resides on the coflow side of the jet shear layers, where flow velocities are relatively low. Internal jet structures are shed from the nozzle, and baroclinic vorticity is observed in the vicinity of the flame. A triple flame is observed at the flame base, due to the gradient in mixture fraction resulting from diffusional premixing of fuel and oxidizer in the incoming flow. Flame stabilization is found to involve premixing and preheating of the fuel and oxidizer streams ahead of the triple flame. The spatial extent of the triple flame is small, such that it is imbedded within the rounded flame base temperature field. The triple flame structure, with its expected rich and lean branches, is modulated by passing vortices, as shown in Figure 2. Peak reaction heat release 

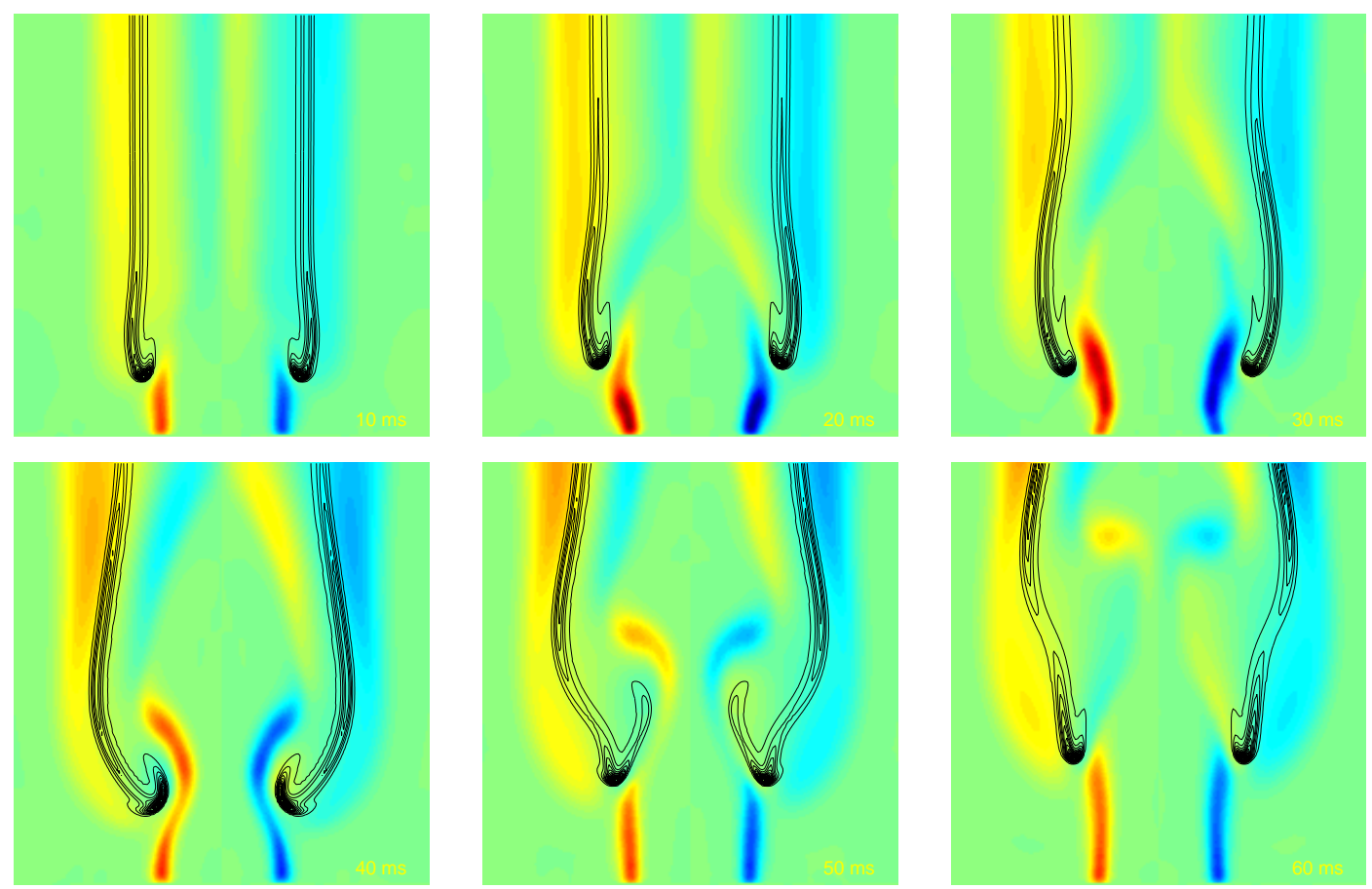

Figure 2: Time evolution of vorticity (colour) and heat release rate (contours) fields at the base of a lifted diffusion jet flame. The interaction of the organized vortical structures emanating from the jet exit with the triple flame structure at the base of the diffusion flame is seen to lead to significant modification of the local flame topology and structure.

rate is observed in the premixed triple flame region, significantly higher than that in the diffusion flame. Given the fine structure of the observed triple flame at the lifted flame base, it is evident that AMR is necessary to provide adequate resolution in this region along with the rest of the domain.

Further studies with different flow and flame conditions, and with realistic chemical kinetics, are necessary to extend and establish the range of validity of these results. Work is also underway to incorporate a stiff time-integration scheme to allow the use of detailed chemical kinetics.

\section{Acknowledgement}

This work was supported by the US Deptartment of Energy (DOE). HNN acknowledges the help of W.T. Ashurst, Sandia National Laboratories-CA, in the numerical formulation.

\section{References}

[1] Devine, K., and Flaherty, J., Appl. Numer. Math., 20:367-386 (1996).

[2] Pember, R.B., Howell, L.H., Bell, J.B., Colella, P., Crutchfield, W.Y., Fiveland, W.A., and Jesse, J.P., Technical Report LBL-38551, Lawrence Berkeley National Laboratory, Berkeley, CA, (1997). 
[3] Najm, H.N., Schefer, R.W., Milne, R.B., Mueller, C.J., Devine, K.D., and Kempka, S.N., Technical Report SAND98-8232, Sandia National Labs., Alb., New Mexico, (1998).

[4] Majda, A., and Sethian, J., Comb. Sci. and Technology, 42:185-205 (1985).

[5] Batchelor, G.K., An Introduction to Fluid Dynamics, Cambridge University Press, England, 1985.

[6] Clark, K., Flaherty, J., and Shephard, M., Appl. Numer. Math., 14 (1994) Eds., Special issue on adaptive methods for partial differential equations.

[7] Gear, C.W., Numerical Initial Value Problems in Ordinary Differential Equations, PrenticeHall, Englewood Cliffs, NJ, 1971.

[8] Berger, M., and Oliger, J., J. Computational Phys., 53:484-512 (1984).

[9] Bell, J.B., Dawson, C.N., and Shubin, G.R., J. Comp. Phys., 74:1-24 (1988).

[10] Bell, J.B., and Colella, P., J. Comp. Phys., 85:257-283 (1989).

[11] Anderson, C.R., J. Comp. Phys., 61:417-444 (1985).

[12] Carrier, J., Greengard, L., and Rokhlin, V., SIAM J. Sci Stat. Comput., 9:669-686 (1988).

[13] Beale, J.T., and Majda, A., Journal of Computational Physics, 58:188-208 (1985).

[14] Beale, J.T., in Computational Fluid Dynamics and Reacting Gas Flows (A. M. M. Luskin. B. Engquist, Ed.), Springer-Verlag, New York, 1988, pp. 19-32, .

[15] Koumoutsakos, P.D., Phd thesis, Graduate Aeronautical Laboratories, California Institute of Technology, Pasadena, CA, (1993).

[16] Winckelmans, G.S., Salmon, J.K., Warren, M.S., Leonard, A., and Jodoin, B., Second International Workshop on Vortex Flows and Related Numerical Methods, Montréal, Canada, (1995).

[17] Monaghan, J.J., Computer Physics Reports, 3:71-124 (1985).

[18] Najm, H.N., and Wyckoff, P.S., Combustion and Flame, 110(1-2):92-112 (1997). 